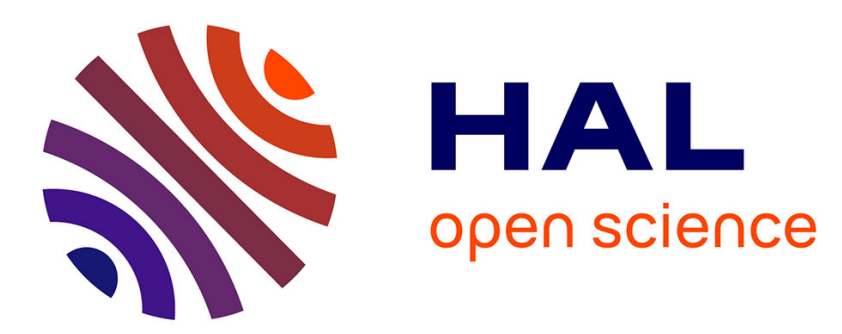

\title{
Identifying Local Arterial Stiffness to Assess the Risk of Rupture of Ascending Thoracic Aortic Aneurysms
}

\author{
Solmaz Farzaneh, Olfa Trabelsi, Bertrand Chavent, Stéphane Avril
}

\section{To cite this version:}

Solmaz Farzaneh, Olfa Trabelsi, Bertrand Chavent, Stéphane Avril. Identifying Local Arterial Stiffness to Assess the Risk of Rupture of Ascending Thoracic Aortic Aneurysms. Annals of Biomedical Engineering, In press. hal-02004901

\section{HAL Id: hal-02004901 https://hal.science/hal-02004901}

Submitted on 2 Feb 2019

HAL is a multi-disciplinary open access archive for the deposit and dissemination of scientific research documents, whether they are published or not. The documents may come from teaching and research institutions in France or abroad, or from public or private research centers.
L'archive ouverte pluridisciplinaire HAL, est destinée au dépôt et à la diffusion de documents scientifiques de niveau recherche, publiés ou non, émanant des établissements d'enseignement et de recherche français ou étrangers, des laboratoires publics ou privés. 


\title{
Identifying local arterial stiffness to assess the risk of rupture of ascending thoracic aortic aneurysms
}

\author{
Solmaz Farzaneh ${ }^{1}$, Olfa Trabelsi ${ }^{1}$, Bertrand Chavent ${ }^{2}$ and Stéphane Avril ${ }^{1 *}$
}

affiliations: ${ }^{1}$ Mines Saint-Étienne, Univ Lyon, Univ Jean Monnet, INSERM, U 1059 Sainbiose, Centre CIS, F - 42023 Saint-Étienne France.

${ }^{2}$ CHU Saint-Étienne, Univ Lyon, Univ Jean Monnet, INSERM, U 1059 Sainbiose, Centre CIS, F - 42023 Saint-Étienne France.

abbreviated title: Identifying local stiffness of ascending aorta aneurysm

correspondence: *Stéphane Avril, Mines Saint-Étienne, Univ Lyon, Univ Jean Monnet, INSERM, U 1059 Sainbiose, Centre CIS, F - 42023 Saint-Étienne France.

Phone: 0477420188, Fax: +33477420000, e-mail: avril@emse.fr 


\begin{abstract}
It was recently submitted that the rupture risk of an ascending thoracic aortic aneurysm (ATAA) is strongly correlated with the aortic stiffness. To validate this assumption, we propose a non-invasive inverse method to identify the patient-specific local extensional stiffness of aortic walls based on gated CT scans. Using these images, the local strain distribution is reconstructed throughout the cardiac cycle. Subsequently, obtained strains are related to tensions, through local equilibrium equations, to estimate the local extensional stiffness at every position. We apply the approach on 11 patients who underwent a gated CT scan before surgical ATAA repair and whose ATAA tissue was tested after the surgical procedure to estimate the rupture risk criterion. We find a very good correlation between the rupture risk criterion and the local extensional stiffness. Finally it is shown that patients can be separated in two groups: a group of stiff and brittle ATAA with a rupture risk criterion above 0.9, and a group of relatively compliant ATAA with a rupture risk below 0.9. Although these results need to be repeated on larger cohorts to impact the clinical practice, they support the paradigm that local aortic stiffness is an important determinant of ATAA rupture risk.
\end{abstract}

keywords: Non-invasive inverse method; Local extensional stiffness; Finite-elements; Patient-specific rupture risk criterion; stretch ratio risk criterion 


\section{$\underline{\text { Introduction }}$}

An ascending thoracic aortic aneurysm (ATAA) is a pathological dilatation of the ascending thoracic aorta. It often grows slowly and usually without symptoms. ATAAs are difficult to detect because it is a silent disease. Small and slow-growing ATAAs may never rupture, but large, fast-growing ATAAs may dissect or rupture, leading to the sudden death of the patient $^{29}$.

Depending on the growth rate and size of a thoracic aortic aneurysm, treatment may vary from watchful waiting to emergency surgery. Preferably, surgery for an ATAA can be planned by replacement of the enlarged aortic segment with synthetic grafts. Excluding the patients with familial genetic disorders such as Marfan syndrome, elective surgical repair of ATAA is recommended for diameter larger than $5.5 \mathrm{~cm}$ or for fast growing aneurysms (growth $>1 \mathrm{~cm}$ per year $)^{24,6,8,9,16}$. The $5.5 \mathrm{~cm}$ diameter criterion is widely acknowledged as an incomplete criterion. The International Registry of Acute Aortic Dissection (IRAD) reported that among 591 type "A" aortic dissections, $59 \%$ had a diameter below $5.5 \mathrm{~cm}$. Studies dedicated to AAA demonstrated that biomechanical factors could reliably complete the diameter criterion ${ }^{19,20}$. Martin et al. ${ }^{28}$ performed a predictive biomechanical analysis of ATAA tissues to assess rupture risk on a patient-specific level. They defined a new rupture risk criterion (the diameter ratio risk) as the ratio between the current diameter of the aneurysm and the rupture diameter. They showed that the diameter ratio risk increases significantly with the physiological elastic modulus of the $\operatorname{artery}^{28}$. Moreover, there is a large interindividual variability of ultimate stress between individuals, which prevents obtaining patient-specific values. Different authors measured geometrical and mechanical properties of ATAA and showed that rupture properties may vary significantly even with tissues having similar elastic properties $^{7,14,15,31,39}$. Our research group proposed a similar rupture risk criterion, namely the stretch ratio risk, defined as the current tissue stretch (under in vivo conditions) and the maximum stretch (at which the tissue ruptures). We derived the stretch ratio risk criterion for a cohort of 31 patients by performing bulge inflation tests on ATAA tissues collected during surgical procedures of these patients. We also derived from these tests the tangent 
elastic modulus of the ATAA tissues and demonstrated that it is strongly correlated to the stretch ratio risk criterion, showing that most of the patients for whom the stretch ratio risk criterion is less than 0.9 have a modulus below $1 \mathrm{MPa}$ whereas patients for whom the stretch ratio risk criterion is greater than 0.9 have a modulus ranging from $1 \mathrm{MPa}$ to $7 \mathrm{MPa}^{15}$.

A natural question is: could the stretch ratio risk criterion be deduced from non invasive measurements of in vivo aortic stiffness? Trabelsi et al. ${ }^{34}$ introduced a methodology to identify the patient-specific material properties of ATAAs by minimization of the difference between model predictions and gated CT measurements of the aneurysm. Furthermore, in an additional study, they estimated volumetric and cross sectional distensibility using preoperative dynamic CT scans and brachial pressures on a cohort of 13 patients. They also characterized the stretch ratio risk criterion and tested its correlation with the aortic stiffness obtained from distensibility measurements. However, only a moderate correlation was found. It was assumed that the lack of significance could be explained as the distensibility stiffness is a global homogeneous property across the whole ATAA whereas the stretch ratio risk criterion is a local rupture property. Moreover it is well-known that the heart motion causes axial strains in the ascending thoracic aorta during cardiac cycles ${ }^{40}$. These axial strains of the ATAA were not taken into account in the distensibility assessment, which may be another source of possible dispersion in the results.

We recently presented a novel methodology, onwards denoted the LESI (local extensional stiffness identification) methodology ${ }^{17}$, to identify local extensional stiffness properties noninvasively, simply employing the gated CT scans and measured systolic and diastolic blood pressures. It was proved that the reconstruction of stiffness is averagely correct and the local heterogeneity can be retrieved using the LESI methodology ${ }^{17}$.

In the present work we show that the local extensional stiffness obtained on a cohort of 11 patients using the LESI methodology correlates well with the stretch ratio risk criterion. 


\section{Materials and methods}

\section{The stretch ratio risk criterion}

ATAA samples were collected for a cohort of 11 patients who underwent elective surgical repair of ATAA at the University Hospital of Saint-Etienne (CHU-SE) in France. Informed consent was obtained according to a protocol approved by the Institutional Review Board of the University Hospital Center of Saint-Etienne. For each sample, a $40 \times 40 \mathrm{~mm}$ square piece was cut and a bulge inflation test was carried out. The bulge inflation test was extensively described in previous publications ${ }^{11,12,15}$.

During the bulge inflation test, the circumferential, $\tau_{1}$, and axial, $\tau_{2}$, components of tension (stress $\times$ thickness), and the circumferential, $\lambda_{1}$, and axial, $\lambda_{2}$, components of CauchyGreen stretch were measured at the center of the sample for each pressure stage, $p(t)$. The stage $t_{\text {physio }}$ at which $\tau_{1}$ equals the average in vivo circumferential stress (according to Laplace's law) was determined according to

$$
\tau_{1}^{t_{p h y s i o}}=\frac{P_{a v} D}{4}
$$

where $P_{a v}$ is the average physiological pressure (mean between diastolic and systolic pressure measured by sphygmomanometry for each patient) and $D$ is the diameter of the aneurysm

measured from the CT scan. We also denote $\lambda_{1}^{t_{\text {physio }}}$ and $\lambda_{2}^{t_{\text {physio }}}$ the circumferential and axial stretches respectively, both measured during the bulge inflation tests at $t_{\text {physio }}$.

Afterwards, the tangent elastic modulus at stage $t_{\text {physio }}$, denoted $E_{\text {in-vitro }}$, was derived as:

$$
E_{\text {in-vitro }}=\lambda_{1}^{t_{\text {physio }}} \frac{d \tau_{1}^{t_{\text {physio }}}}{d \lambda_{1}^{t_{\text {physio }}}}
$$

At the last stage of the bulge inflation test, when the ATAA sample burst, the rupture stretch and stress, measured in the direction perpendicular to the crack splitting the sample in two halves, were respectively denoted $\lambda_{\text {rup }}$ and $\sigma_{\text {rup }}$. 
Finally the stretch ratio risk criterion was defined as:

$$
\gamma_{\text {stretch }}=\sqrt{\left(\frac{\lambda_{1}^{t_{\text {physio }}}}{\lambda_{\text {rup }}}\right)^{2} \cos ^{2} \theta+\left(\frac{\lambda_{2}^{t_{\text {physio }}}}{\lambda_{\text {rup }}}\right)^{2} \sin ^{2} \theta}
$$

where $\theta$ is the angle between $e_{\theta}$ and $e_{2}$ as shown in Fig. 2 of Duprey et al. ${ }^{15} \cdot \gamma_{\text {stretch }}$ takes a value between 0 and 1 .

The clinical and biomechanical parameters of the 11 patients are reported in Table 1.

\section{The LESI methodology}

\section{Data acquisition}

Gated CT scans were obtained for the 11 patients prior to ATAA surgical repair. Ten phases were acquired throughout the cardiac cycle for each patient and they were employed to reconstruct and track the geometrical variations of the whole aorta. Semi-automatic segmentation of the CT scans was achieved using MIMICS (v.10.01, Materialise NV). The obtained three-dimensional (3D) surface of the aorta was exported for each phase in STL format. The Vascular Modeling Toolkit (VMTK, Orobix, Bergamo, Italy; www.vmtk.org) ${ }^{1}$ was used to generate a structural mesh of the exported geometries. In order to run VMTK and to obtain the parametric coordinates of the surface, aortic surfaces were cut at all phases by the same cross sectional planes to determine a larger domain than the final segment of interest. An identical smoothing factor was applied at all phases. To identify the systolic and diastolic phases, the luminal volumes of all phases were compared. The systolic phase was defined as the largest volume and the diastolic phase as the smallest volume. The variation of cross sectional area between diastole and systole was used to derive the arterial distensibility and to deduce the extensional stiffness property based on in-vivo distensibility, denoted $E_{\text {in-vivo }}$. Results are reported in Table 1 and were derived from a previous analysis ${ }^{35}$.

\section{Foundation of the LESI methodology}

Each reconstructed aortic geometry was meshed in such a way that each node represented the position of the same material point at each phase of the cardiac cycle. Thus, a structural 
mesh is required, with an identical number of elements and nodes at all phases. Therefore, a mesh morphing function between the geometries of each phase was reconstructed and applied. VMTK was used to generate the structural mesh from segmented geometries (STL files). VMTK is based on the calculation of centerlines and decomposition of the surface into existing branches and mapping each branch onto template parametric cylindrical coordinates. VMTK generates consistent parameterizations over a wide range of bifurcating geometries. The procedure to obtain parametric coordinates by VMTK, is explained in detail in Farzaneh et al. ${ }^{17}$. In order to track the same material points at all phases throughout the cardiac cycle, we assumed anatomical references, namely the left subclavian artery, the brachiocephalic artery, the left common carotid artery and the coronary artery branches. Eventually, the obtained data from VMTK were postprocessed in Matlab to extract an accurate mesh using the longitudinal and circumferential metrics. Moreover, twisting ocurring during aortic wall motions ${ }^{40}$ was also corrected by deriving the time variations of the circumferential coordinate of coronary arteries in the space of parametric coordinates obtained from VMTK and subtracting this value from the circumferential coordinate of the assumed reference phase ${ }^{17}$.

Any periodic function (such as material coordinates in each phase of a cardiac cycle, $x(t))$ can be expressed as a summation of sine and cosine functions of varying frequency. Therefore, using the structural mesh of all ten phases, a discrete Fourier transform was employed such as

$$
x(t)=a_{0}+\sum_{n=1}^{\infty}\left(a_{n} \cos (n f t)+b_{n} \sin (n f t)\right)
$$

where $a_{0}$ is the Direct current (DC) term (average) and $f$ is the fundamental frequency. Strains in longitudinal and circumferential directions were obtained on the average geometry (defined by using DC terms of all nodal positions) by applying displacements and torsion at each node corresponding to the fundamental term obtained from the Fourier transform in Eq. 4. As the fundamental term is complex, the real and the imaginary parts are applied separately at the corresponding nodes on the average geometry using the finite element method (FEM). Eventually the reconstructed strain components were also complex ${ }^{17}$. 
Therefore the magnitude of strains in the longitudinal and circumferential directions could be obtained ${ }^{17}$ such as

$$
\begin{aligned}
& \varepsilon_{1}=\left(\varepsilon_{1}^{\mathrm{r} 2}+\varepsilon_{1}^{\mathrm{im}^{2}}\right)^{1 / 2} \\
& \varepsilon_{2}=\left(\varepsilon_{2}^{\mathrm{r} 2}+\varepsilon_{2}^{\mathrm{im}}\right)^{1 / 2}
\end{aligned}
$$

where superscripts " $r$ " and " $i m$ " indicate real and imaginary contributions, respectively. It is well-known that the aortic tissue is globally anisotropic and nonlinear, but here its mechanical behaviour was linearized in the physiological range of strains between diastole and systole, and anisotropic effects was neglected in this range. The constitutive equations reduced to Hooke's law and the plane stress assumption was considered ${ }^{22}$. The membrane tensions, $\tau$, were written such as

$$
\begin{aligned}
& \tau_{1}(t)=\tau_{1}^{0}+Q\left(\varepsilon_{1}(t)+\nu \varepsilon_{2}(t)\right) \\
& \tau_{2}(t)=\tau_{2}^{0}+Q\left(\nu \varepsilon_{1}(t)+\varepsilon_{2}(t)\right)
\end{aligned}
$$

where $Q$ is the extensional stiffness and $\nu$ is the Poisson's ratio. $\tau_{1}^{0}$ and $\tau_{2}^{0}$ are the components, in the local coordinate system ( 1 is for local circumferential direction and 2 is for local axial direction), of the membrane stresses (tensions) resulting from the average pressure applied onto the average aortic geometry. A FE stress analysis was implemented to derive $\tau_{1}^{0}$ and $\tau_{2}^{0}$ by applying the average pressure $P_{0}$ on the average geometry using the approach proposed by Joldes et al. ${ }^{25}$.

It was assumed that each element of the arterial wall is a finite sector of an ellipsoidal membrane with radii $r_{1}$ and $r_{2}$ in both circumferential and longitudinal directions, respectively. So, in the framework of these assumptions, local equilibrium equations were written as

$$
P(t)=\frac{\tau_{1}(t)}{r_{1}(t)}+\frac{\tau_{2}(t)}{r_{2}(t)}
$$

where $P(t)$ is the applied pressure. Thus linking Eqs. 6 with 7, we obtained the extensional stiffness as

$$
Q=\frac{\Delta P+\frac{\tau_{1}^{0} \Delta r_{1}}{\left(r_{1}^{0}\right)^{2}}+\frac{\tau_{2}^{0} \Delta r_{2}}{\left(r_{2}^{0}\right)^{2}}}{\frac{\varepsilon_{1}(t)+\nu \varepsilon_{2}(t)}{r_{1}^{0}}+\frac{\nu \varepsilon_{1}(t)+\varepsilon_{2}(t)}{r_{2}^{0}}}
$$

where $\Delta P=\left(P_{\text {sys }}-P_{\text {dias }}\right) / 2$. 
To obtain $r_{1}$ and $r_{2}$, we developed a method based on the principle of virtual work as introduced by Bersi et al. ${ }^{5}$ and Farzaneh et al. ${ }^{17}$.

\section{Numerical implementation of the LESI methodology}

For each aorta, the Abaqus FE software ${ }^{21}$ was employed to derive strains from the nodal displacements obtained with the Fourier transform. For each FE analysis, an orientation user subroutine (ORIENT) was employed to assign local material directions and to record the results in the local coordinate system. Each geometry was a non-perfectly cylindrical geometry so that the radial direction (normal to the artery) was defined as the outward normal direction to each element, the axial direction was defined as the direction parallel to the luminal centerline in the direction of the blood flow, and the circumferential direction was perpendicular to both previously defined directions. To calculate and visualize the local extensional stiffness obtained from Eq. 8, a user material subroutine (UMAT) and a user-defined external databases (UEXTERNALDB) were coupled with Abaqus ${ }^{21}$.

In summary, as shown in Fig. 1, we have four types of FE analyses to be performed in our methodology:

1. Strain calculation: this was a displacement driven finite element analysis. Displacements derived from $\mathrm{CT}$ images were applied to each node of the mesh in the reference geometry.

2. Radii of curvature calculation: this was again a displacement driven finite element analysis. A displacement normal the luminal surface was applied at each node. The resulting strain fields equaled the local curvature fields, which permitted a direct evaluation of the local radii of curvature.

3. Calculation of average tensions: using the average geometry, an average pressure was applied on the lumen. In the inlet and outlet nodes, we assigned displacements assessed using the dynamic CT images. 
4. Validation study: the difference between systolic and diastolic pressures (pulsed pressure) was applied on the inner luminal surface. Nodal displacements of inlets and outlets were zeroed.

\section{Numerical validation of the LESI methodology}

Two different material behaviors were considered to perform numerical validations: linear isotropic elasticity and non-linear anisotropic hyperelasticity. The reconstructed stiffness was in good agreement with the reference, especially local heterogeneities could be well retrieved $^{17}$.

\section{Statistical analysis}

Three regions were defined in the ascending thoracic aorta of each patient:

1. ATAA region 1 is the whole ascending thoracic aorta.

2. ATAA region 2 is the segment of ascending thoracic aorta which was removed during the surgical procedure.

3. ATAA region 3 is the square sample of $40 \times 40 \mathrm{~mm}$ which was cut for the bulge inflation test.

As the tests of normality show that the data is non-normal, for the sake of statistical analysis, we derived the median of the identified extensional stiffness across each of the 3 ATAA regions defined above, denoted respectively $Q_{1}, Q_{2}$ and $Q_{3}$. We also derived the value of the identified extensional stiffness at the center of ATAA region 3 , denoted $Q_{4}$. The derived median values are very close to mean values.

Patients were divided in two groups based on their $\gamma_{\text {stretch }}$ value, as this appears to be the relevant criterion to estimate the risk of rupture ${ }^{15}$. Then, $Q_{1}, Q_{2}, Q_{3}$ and $Q_{4}$ for each group were analyzed as median and the 25 th and 75 th percentiles using boxplots. Statistical pairwise comparisons were made across the 2 groups for each of the 4 material parameters. 
The $\mathrm{P}$-value was derived for each quantity. Significant differences (denoted by *) were identified for comparisons with $\mathrm{p}<0.05$.

We also searched for correlations between stiffness values identified in vivo $\left(Q_{1}, Q_{2}\right.$, $Q_{3}$ and $\left.Q_{4}\right)$ and the properties characterized in vitro, including $\sigma_{\text {rup }}, E_{\text {in-vitro }}$ and $\gamma_{\text {stretch }}$. These correlations are shown in Fig. 2 by curve fitting with regression. The Pearson productmoment correlation coefficient was also derived to assess the dependence between each couple of quantities.

\section{$\underline{\text { Results }}$}

The median values of extensional stiffness $\left(Q_{1}, Q_{2}, Q_{3}\right.$ and $\left.Q_{4}\right)$ are reported in Table 2 . We report the Pearson product-moment correlation coefficient, $\rho$, between stiffness properties identified in vivo $\left(Q_{1}, Q_{2}, Q_{3}\right.$ and $\left.Q_{4}\right)$ and the properties characterized in vitro, including $\sigma_{\text {rup }}, E_{\text {in-vitro }}$ and $\gamma_{\text {stretch }}$ in Table 3 . We found a large correlation between $Q_{4}$ and $\gamma_{\text {stretch }}$ $(\rho=0.89)$. Other extensional stiffness properties identified in vivo also show some correlation with $\gamma_{\text {stretch}}$, but the best correlation was found for $Q_{4}$, which is the identified stiffness at the center of ATAA region 3. Note that no correlation was found between $\sigma_{\text {rup }}$ and the identified stiffness properties.

Patients were divided in two groups based on their stretch ratio risk index ${ }^{15}$ :

- group 1 composed of 5 patients with a large stretch ratio risk index: $\gamma_{\text {stretch }}>0.9$.

- group 2 composed of 6 patients with a relatively small stretch ratio risk index: $\gamma_{\text {stretch }}<$ 0.9 .

The stress-stretch curves of their ATAA sample derived from the bulge inflation test are shown in Fig. 3. Solid lines indicate the state of stress and stretch undergone by the aortic tissue in vivo. The same maximum stress is always reached in the circumferential and axial directions of the tissue as the bulge inflation test induces a state of nearly equibiaxial tension. However, anisotropy manifests more evidently for some patients in the maximum stretches, 6/11 patients having a larger circumferential stretch and 5/11 a larger axial stretch. 
In Fig. 4, we show the reconstructed geometry for all patients. Remarkably, Fig. 4-h is a patient with Marfan syndrome and in Fig. 4-d the patient had a bovine arch which is a rare malformation often related to ATAA.

In Fig. 5, we show the boxplots of median extensional stiffness values for both groups of patients. A boxplot is shown for each stiffness value $\left(Q_{1}, Q_{2}, Q_{3}\right.$ and $\left.Q_{4}\right)$. A significant difference between both groups is shown for the $Q_{4}$ value with a p-value of 0.027 . The identified stiffness distribution obtained with the LESI methodology is shown for each patient in regions 1, 2 and 3 (Fig. 6).

\section{$\underline{\text { Discussion }}$}

In this study the LESI methodology ${ }^{17}$ was employed to reconstruct noninvasively the stiffness distribution of the ascending thoracic aorta of 11 patients based on their preoperative gated CT scans. For each patient, the stretch ratio risk criterion was also characterized postoperatively using the bulge inflation test ${ }^{15}$. A very good correlation was found between the extensional stiffness and the stretch ratio risk index. Finally it was shown that patients can be separated in two groups: a group of stiff and brittle ATAA with a rupture risk criterion above 0.9, and a group of relatively compliant ATAA with a rupture risk below 0.9. This shows that the in vivo arterial stiffness could be used to derive the stretch ratio risk index. The cut-off value of 0.9 was proposed in a previous paper ${ }^{15}$ in which we had tested ATAA samples coming from over 30 patients. The statistical analysis nicely showed that the group of ATAA with rupture risk below 0.9 and the group with rupture risk above 0.9 were two different groups. In the current paper, only 11 patients are analyzed as only these 11 patients had both a gated CT scan and a bulge inflation test.

Here we used gated CT to reconstruct the local aortic stiffness. Other imaging modalities may be possible such as ultrasounds ${ }^{37,40}$ or magnetic resonance imaging ${ }^{2}$. 


\section{Rupture risk criterion}

Multiple studies have been developed to define a reliable rupture risk criterion for ATAA ${ }^{28,31,15}$ and $\mathrm{AAA}^{38}$. For instance, Martin et al. ${ }^{28}$ defined a criterion, named the diameter ratio risk, which is the ratio between the current diameter of the aneurysm and the rupture diameter. They showed that the diameter risk increases significantly with the physiological elastic modulus of the artery. Commonly, rupture risk is also estimated as the ratio between current (or physiological) and ultimate stress. However, wall stress estimation suffers from uncertainties in wall thickness measurement. Moreover, there is a large interindividual variability of ultimate stress between individuals, which prevents obtaining patient-specific values. Different authors measured geometrical and mechanical properties of ATAA and showed that rupture properties may vary significantly even with tissues having similar elastic properties $7,14,15,31,39$. Moreover, another disadvantage of stress based rupture index is that current stress induced by normal blood pressures is usually far from the ultimate stress. Indeed, rupture is often an accidental event during which there is a sudden rise of pressure and stress in the wall. There is evidence showing that dissections are preceded by a specific severe exertional or emotional event $^{16}$. The stretch ratio risk index ${ }^{15}$, defined as the current tissue stretch (under in vivo conditions) and the maximum stretch (at which the tissue ruptures), appears very promising as it is strongly correlated with the local tissue stiffness which can be obtained with the LESI methodology. Conversely our results showed that there is no correlation between the strength of ATAA samples and their in vivo stiffness.

In addition, to examine various criteria of the local failure of AAA, Volokh et al. ${ }^{38}$ developed an experimental model including a failure description. They analyzed various states of the biaxial tension of the AAA material and evaluated the strain energy, the maximum principal stretch and stress, the maximum shear stress and von Mises stress criteria of the local failure. They concluded that the von Mises stress showed a larger variability compared to the strain energy. It could be interesting to define rupture risk criterion based on maximum stored strain energy as it would combine both stress and strain contributions, but apart from Volokh et al., nobody really investigated this way further. 


\section{Regional variations of stiffness across ATAA}

A major conclusion of the current study is that it is important to identify local stiffness properties and not a global stiffness property based on distensibility analysis for instance ${ }^{35}$, as rupture is a local phenomenon. Over the past decades the biomechanical properties of the aorta have been widely investigated ${ }^{14,15,31,35,39}$, but the material properties were usually characterized globally and homogeneously across ATAA. A few studies considered regional variations of material properties across ATAA. Iliopoulos et $\mathrm{al}^{23}$ stated that circumferential specimens were stiffer than longitudinal ones in the posterior and left lateral. Furthermore they found no significant regional variations of aortic stiffness in the circumferential direction. Ferrara et al. ${ }^{18}$ supported these results and investigated mechanical properties of human ascending aortas for a cohort of patients taking into account regional, directional, aging and gender variations. They showed that regardless of age and gender, the mean elastic modulus was larger in the circumferential direction. Moreover, aging reduced the mean elastic modulus and male had higher mean elastic modulus comparing to female. Choudhury et al. ${ }^{7}$ compared stiffness and material properties of ATAA at different angular positions and showed that the outer and the inner curvatures are the most stiffest and elastic quadrants respectively. Recently, digital image correlation (DIC) was used to investigate the local material properties of excised aortic samples ${ }^{3,11,12}$. Inverse methods were developed to process DIC measurements and reconstruct the regional variations of material properties.

Regional variations of material properties are attributed to remodeling of the arterial wall due to damage of elastic fibers. A typical wall histopathology, involving ECM breakdown (with disrupted elastin networks), vSMC loss co-localized with areas of mucoid degeneration, is common to ATAA whatever their aetiologies ${ }^{29}$. When elastin networks are disrupted, a larger fraction of collagen fibers need to be produced to resist to the wall stress ${ }^{36}$. This manifests with aortic stiffening and reduced extensibility (stretch based rupture risk), which are the two markers for which we found correlations in our study. Sassani et al ${ }^{30}$ demonstrated a large distribution of fiber orientations spanning from circumferential to longitudinal directions, which was also supported by Sokolis et al. ${ }^{33}$ showing that ATAA had no effect on the 
tissue strength. However, this can cause stiffening and extensibility reduction, confirmed by histological observations of elastin degradation and increase of collagen mass fractions. It could be interesting also to check the possible correlation of these regions with local concentration of wall shear stress induced by the blood flow ${ }^{27,10}$.

\section{Limitations}

Several limitations in this work can be discussed.

- The residual stresses existing in the ATAA wall were neglected in this work. More specifically, we report that a major limitation of our study is that we do not consider layer specific material and rupture properties of ATAA. As shown by Sokolis et al. ${ }^{32}$ residual stretches may vary strongly from one layer to another, and depend also on circumferential positions. It was also shown that failure stretches correlate positively with residual stretches ${ }^{13}$. Therefore, layer-specific properties are important aspects that should be integrated in the future to rupture risk estimation of ATAAs. In addition, it was assumed that the arterial wall behaves as an isotropic and linearly elastic material within the systolic-diastolic range. Therefore, the isotropy assumption here delivers a combination of the axial and circumferential extensional stiffnesses, which may be different. However, if there was a significant difference between the axial and circumferential extensional stiffnesses, the extensional stiffness identified here would be closer to the circumferential one, as shown in a previous study ${ }^{17}$. Involving the local curvatures in Eq. 8 puts most of the weight on circumferential effects, as the axial radius of curvature is significantly higher than the circumferential one.

- The LESI approach stands on local equilibrium equations obtained based on the principle of virtual work, similar to the ones derived by bersi et al. ${ }^{5}$. Therefore, equivalent to the generalized Laplace's law, it considers the local equilibrium between pressures and tensions in a membrane, meaning that the aortic wall experience no shear through the thickness. This may not be a realistic assumption in the regions near the branches 
which were excluded in this analysis.

- Eq. 8 involves average tensions existing in the aorta due to the action of the average blood pressure and geometrical nonlinearity associated to finite deformations ${ }^{4}$. These tensions were approximated using the approach of Joldes et al. ${ }^{25}$. This approach consists in achieving a linear elastic stress analysis on the same structure as the aorta, assigning a very large elastic modulus. The approach is valid for membrane structures. The membrane assumption was previously shown to be a good approximation for elastostatics of aortic aneurysms ${ }^{26}$.

- A final limitation is that $\gamma_{\text {stretch }}$ evaluates the ex vivo rupture risk, not the in vivo one (for instance, the role of vSMC tone or surrounding tissues may be important in vivo).

\section{Acknowledgements}

The authors are grateful to the European Research Council for grant ERC-2014-CoG BIOLOCHANICS.

\section{Conflict of interest}

The authors have no conflict of interest to declare concerning the content of this manuscript.

\section{References}

1. Antiga, L. and D. Steinman. Robust and objective decomposition and mapping of bifurcating vessels. IEEE Trans Med Imaging 23(6):704-13, 2004.

2. Arani, A., S. Arunachalam, I. Chang, F. Baffour, P. Rossman, K. Glaser, J. Trzasko, K. McGee, A. Manduca, M. Grogan, A. Dispenzieri, R. Ehman, and P. Araoz. Cardiac mr elastography for quantitative assessment of elevated myocardial stiffness in cardiac amyloidosis. J Magn Reson Imaging. 46(5):1361-1367, 2017. 
3. Avril, S., P. Badel, and A. Duprey. Anisotropic and hyperelastic identification of in vitro human arteries from full-field optical measurements. Journal of biomechanics 43:2978-85, 2010.

4. Baek, S., R. Gleason, K. Rajagopal, and J. Humphrey. Theory of small on large: potential utility in computations of fluid--solid interactions in arteries. Comput Methods Appl Mech Eng 196:3070-78, 2007.

5. Bersi, M., C. Belliniand, P. D. Achille, J. Humphrey, K. Genovese, and S. Avril. Novel methodology for characterizing regional variations in the material properties of murine aortas. Journal of biomechanical engineering 138:071005, 2016.

6. Chau, K. and J. Elefteriades. Natural history of thoracic aortic aneurysms: size matters and plus moving beyond size. Prog Cardiovasc Dis 56(1):74-80, 2013.

7. Choudhury, N., B. Olivier, R. Leonie, T. Dominique, C. Raymond, and B. J. et al. Local mechanical and structural properties of healthy and diseased human ascending aorta tissue. Cardiovasc Pathol 18:83-91, 2009.

8. Coady, M., J. Rizzo, G. Hammond, G. Kopf, and J. Elefteriades. Surgical intervention criteria for thoracic aortic aneurysms: a study of growth rates and complications. Ann Thorac Surg 67(6):1922-6, 1999.

9. Coady, M., J. Rizzo, G. Hammond, D. Mandapati, U. Darr, G. Kopf, and J. Elefteriades. What is the appropriate size criterion for resection of thoracic aortic aneurysms? J Thorac Cardiovasc Surg 113(3):476-91, 1997.

10. Condemi, F., S. Campisi, M. Viallon, T. Troalen, G. Xuexin, A. Barker, M. Markl, P. Croisille, O. T. C. Cavinato, A. Duprey, and S. Avril. Fluid- and biomechanical analysis of ascending thoracic aorta aneurysm with concomitant aortic insufficiency. Ann Biomed Eng. 45(12):2921-2932, 2017. 
11. Davis, F., Y. Luo, S. Avril, A. Duprey, and J. Lu. Pointwise characterization of the elastic properties of planar soft tissues: application to ascending thoracic aneurysms. Biomechanics and modeling in mechanobiology 14:967-78, 2015.

12. Davis, F., Y. Luo, S. Avril, A. Duprey, and J. Lu. Local mechanical properties of human ascending thoracic aneurysms. Journal of the mechanical behavior of biomedical materials $61: 235-49,2016$.

13. Deveja, R., D. Iliopoulos, E. Kritharis, D. Angouras, D. Sfyris, S. Papadodima, and D. Sokolis. Effect of aneurysm and bicuspid aortic valve on layer-specific ascending aorta mechanics. Ann Thorac Surg 106(6):1692-1701, 2018.

14. Duprey, A., K. Khanafer, M. Schlicht, S. Avril, D. Williams, and R. Berguer. In vitro characterisation of physiological and maximum elastic modulus of ascending thoracic aortic aneurysms using uniaxial tensile testing. Eur J Vasc Endovasc Surg 39(6):700-7, 2010.

15. Duprey, A., O. Trabelsi, M. Vola, J. Favre, and S. Avril. Biaxial rupture properties of ascending thoracic aortic aneurysms. Acta Biomater 42:273-85, 2016.

16. Elefteriades, J. and E. Farkas. Thoracic aortic aneurysm clinically pertinent controversies and uncertainties. J Am Coll Cardiol 55(9):841-57, 2010.

17. Farzaneh, S., O. Trabelsi, and S. Avril. Inverse identification of local stiffness across ascending thoracic aortic aneurysms. Biomechanics and Modeling in Mechanobiology , 2018.

18. Ferrara, A., S. Morganti, P. Totaro, A. M. A, and F. Auricchio. Human dilated ascending aorta: Mechanical characterization via uniaxial tensile tests. J Mech Behav Biomed Mater $53: 257-71,2016$.

19. Fillinger, M., S. Marra, M. Raghavan, and F. Kennedy. Prediction of rupture risk in abdominal aortic aneurysm during observation: wall stress versus diameter. J Vasc Surg 37(4):724-32, 2003. 
20. Vande, G. J. P., E. D. Martino, A. Bohra, M. Makaroun, and D. Vorp. A biomechanicsbased rupture potential index for abdominal aortic aneurysm risk assessment: demonstrative applicatio. Ann N Y Acad Sci 1085:11-21, 2006.

21. Hibbit, Karlson, and Sorensen. Abaqus-Theory manual, 6.11-3 edition, 2011.

22. J. Humphrey Cardiovascular solid mechanics: cells, tissues, and organs. Springer Science \& Business Media, 2013.

23. Iliopoulos, D., E. Kritharis, A. Giagini, and D. S. S.A. Papadodima. Ascending thoracic aortic aneurysms are associated with compositional remodeling and vessel stiffening but not weakening in age-matched subjects. J Thorac Cardiovasc Surg 137(1):101-9, 2009.

24. Johansson, G., U. Markström, and J. Swedenborg. Ruptured thoracic aortic aneurysms: a study of incidence and mortality rates. J Vasc Surg 21(6):985-8, 1995.

25. Joldes, G., K. Miller, A. Wittek, and B. Doyle. A simple, effective and clinically applicable method to compute abdominal aortic aneurysm wall stress. J Mech Behav Biomed Mater 58:139-48, 2016.

26. Lu, J., X. Zhou, and M. Raghavan. Inverse elastostatic stress analysis in pre-deformed biological structures: demonstration using abdominal aortic aneurysms. J Biomech 40:693$6,2007$.

27. Malvindi, P., S. Pasta, G. Raffa, and S. Livesey. Computational fluid dynamics of the ascending aorta before the onset of type a aortic dissection. European Journal of CardioThoracic Surgery 51(3):597-99, 2016.

28. Martin, C., W. Sun, T. Pham, and J. Elefteriades. Predictive biomechanical analysis of ascending aortic aneurysm rupture potential. Acta Biomater 9(12):9392-400, 2013.

29. Michel, J., G. Jondeau, and D. Milewicz. From genetics to response to injury: vascular smooth muscle cells in aneurysms and dissections of the ascending aorta. Cardiovascular Research 114:578-589, 2018. 
30. Sassani, S., S. Tsangaris, and D. Sokolis. Layer- and region-specific material characterization of ascending thoracic aortic aneurysms by microstructure-based models. J Biomech 48(14):3757-65, 2015.

31. Smoljkić, M., H. Fehervary, P. V. den Bergh, A. Jorge-Pẽ̃́sas, L. Kluyskens, S. Dymarkowski, P. Verbrugghe, B. Meuris, J. V. Sloten, and N. Famaey. Biomechanical characterization of ascending aortic aneurysms. Biomech Model Mechanobiol. 16(2):705-720, 2016 .

32. Sokolis, D. Effects of aneurysm on the directional, regional, and layer distribution of residual strains in ascending thoracic aorta. J Mech Behav Biomed Mater 46:229-43, 2015.

33. Sokolis, D., E. Kritharis, A. Giagini, K. Lampropoulos, S. Papadodima, and D. Iliopoulos. Biomechanical response of ascending thoracic aortic aneurysms: association with structural remodelling. Comput Methods Biomech Biomed Engin 15(3):231-48, 2012.

34. Trabelsi, O., A. Duprey, J. Favre, and S. Avril. Predictive models with patient specific material properties for the biomechanical behavior of ascending thoracic aneurysms. Ann Biomed Eng 44(1):84-98, 2016.

35. Trabelsi, O., M. Gutierrez, S. Farzaneh, A. Duprey, and S. Avril. A noninvasive technique for ATAA rupture risk estimation. Journal of Biomechanics p. doi.org/10.1016/j.jbiomech.2017.11.012, 2017.

36. Valentín, A., J. Humphrey, and G. Holzapfel. A multi-layered computational model of coupled elastin degradation, vasoactive dysfunction, and collagenous stiffening in aortic aging. Ann Biomed Eng 39(7):2027-45, 2011.

37. van Disseldorp, E., N. Petterson, M. Rutten, F. van de Vosse, M. van Sambeek, and R. Lopata. Patient specific wall stress analysis and mechanical characterization of abdominal aortic aneurysms using 4D ultrasound. Eur J Vasc Endovasc Surg 52:635-42, 2016. 
38. Volokh, K. Comparison of biomechanical failure criteria for abdominal aortic aneurysm. J Biomech. 43(10):2032-4, 2010.

39. Vorp, D., B. Schiro, M. Ehrlich, T. Juvonen, M. Ergin, and B. Griffith. Effect of aneurysm on the tensile strength and biomechanical behavior of the ascending thoracic aorta. Ann Thorac Surg 75:1210-14, 2003.

40. Wittek, A., K. Karatolios, C. Fritzen, J. Bereiter-Hahn, B. Schieffer, R. Moosdorf, S. Vogt, and C. Blase. Cyclic three-dimensional wall motion of the human ascending and abdominal aorta characterized by time-resolved three-dimensional ultrasound speckle tracking. Biomech Model Mechanobiol 15(5):1375-88, 2016. 


\section{List of Figures}

1 Flowchart of the LESI methodology . . . . . . . . . . . . . . . . 23

2 Regression of stiffness versus biomechanical parameters . . . . . . . . . . 24

3 Stress-stretch curves . . . . . . . . . . . . . . . . . . . . . . . . . . . . . . . . . . . . . . . . .

4 Segmented geometries of aorta . . . . . . . . . . . . . 26

5 Boxplots of both groups of patients . . . . . . . . . . . . . . 27

6 Extensional stiffness distribution . . . . . . . . . . . . . . 28 


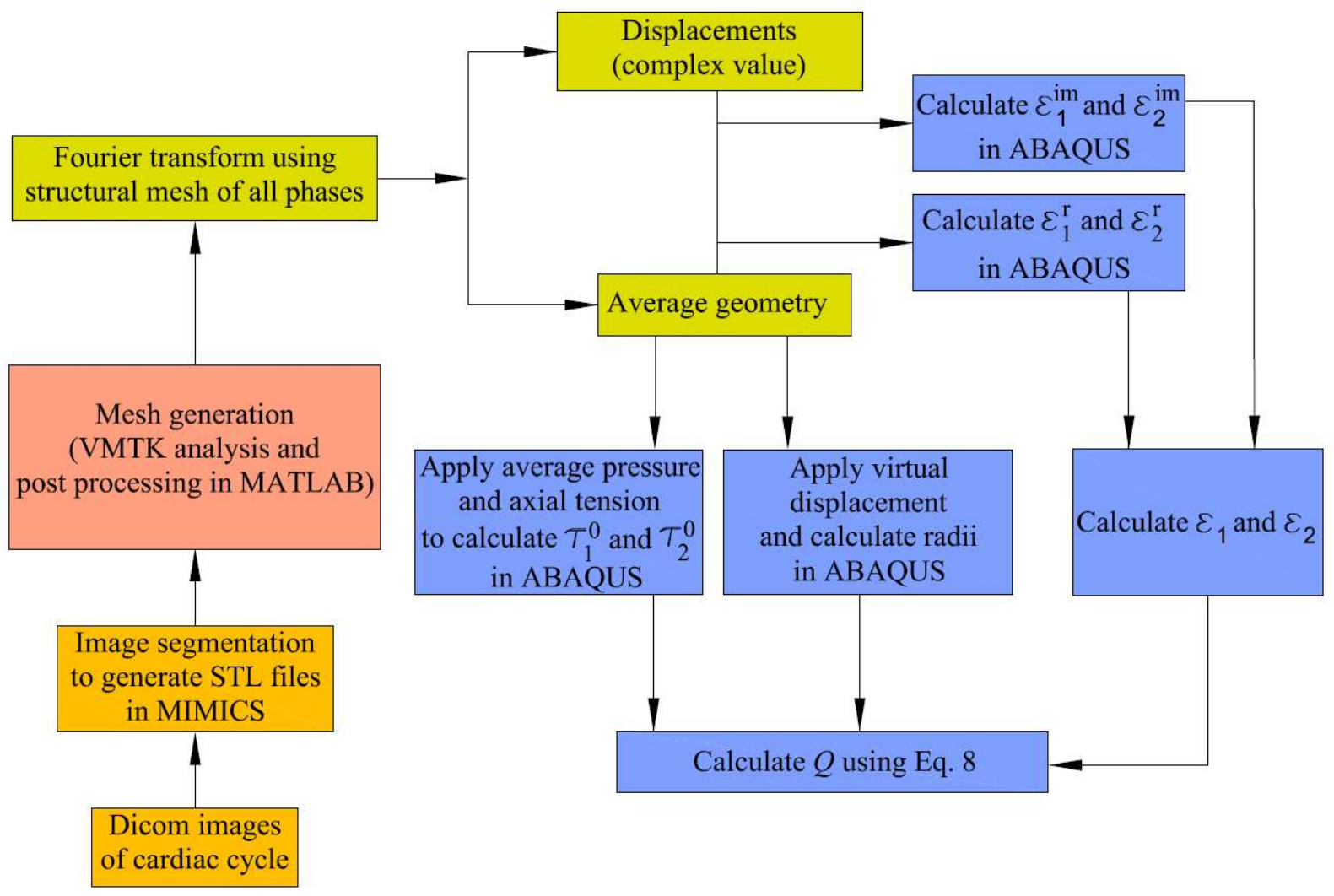

Figure 1: Flowchart of the LESI methodology for local extensional stiffness identication. 


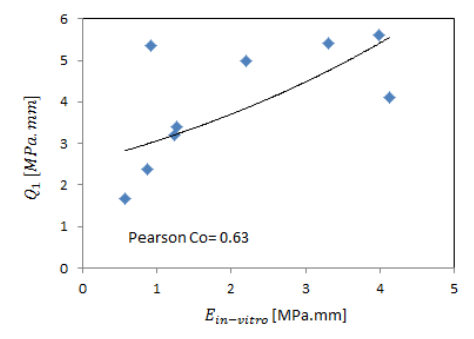

(a)

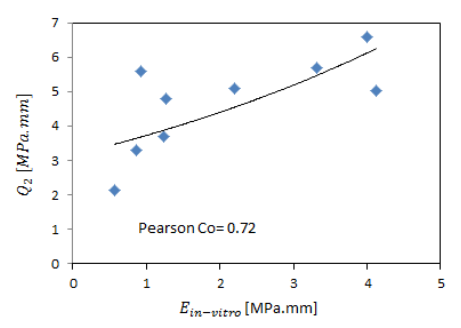

(d)

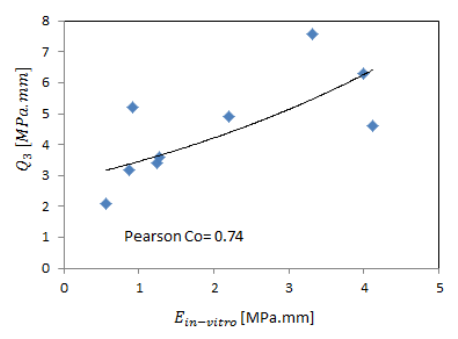

(g)

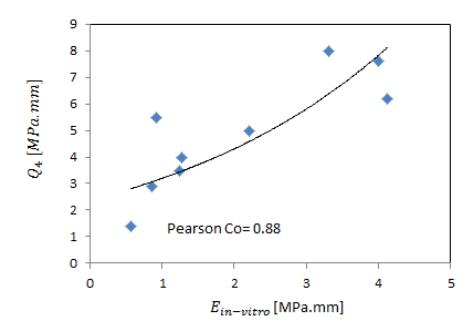

(j)

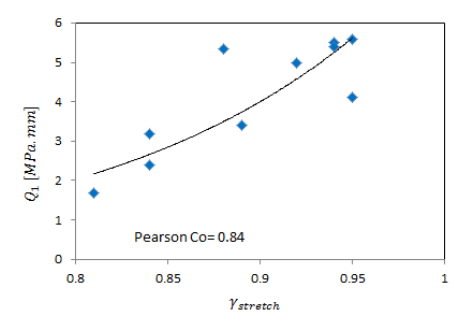

(b)

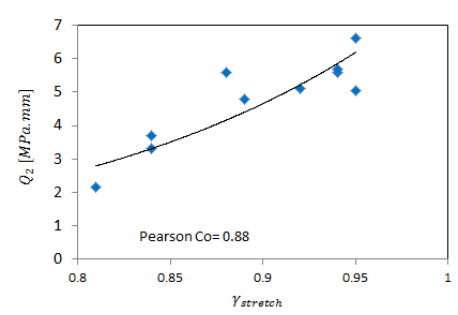

(e)

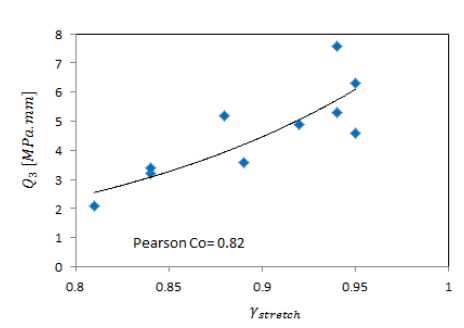

(h)

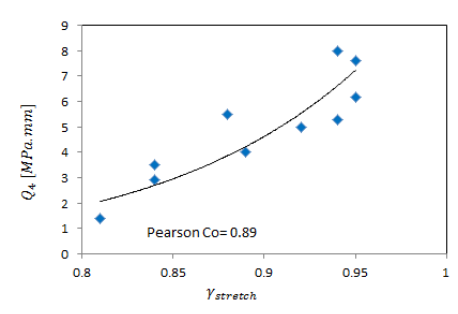

$(\mathrm{k})$

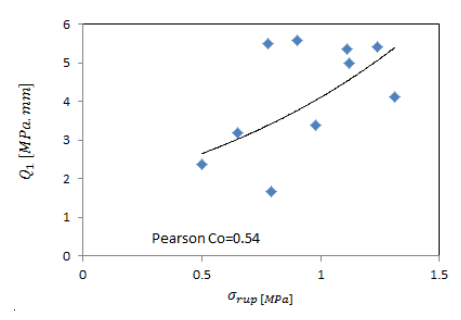

(c)

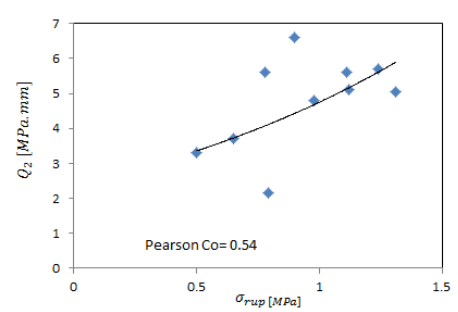

(f)

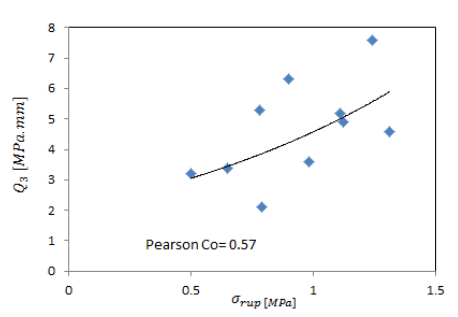

(i)

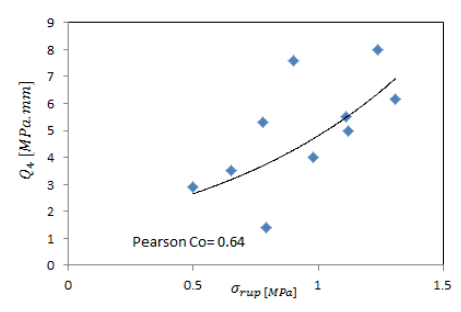

(1)

Figure 2: Regression of $Q_{1}, Q_{2}, Q_{3}$, and $Q_{4}$ versus stretch ratio risk criterion, rupture stress and in - vitro stiffness ${ }^{15}$ of patients. 


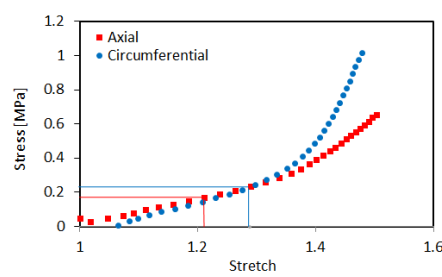

(a) patient 1

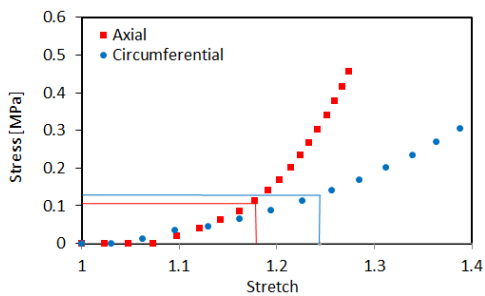

(d) patient 4

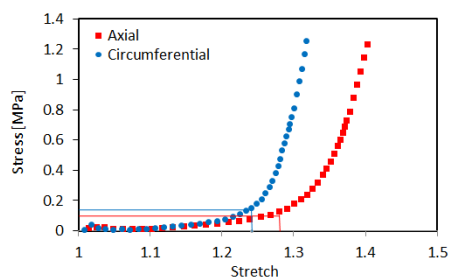

(g) patient 7

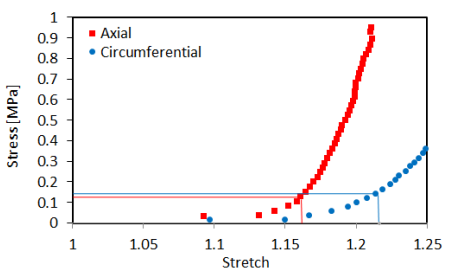

(j) patient 10

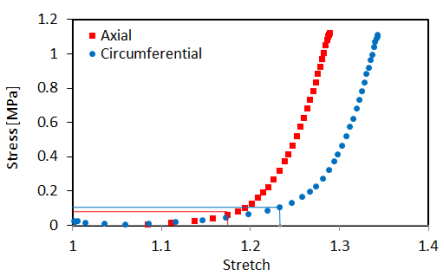

(b) patient 2

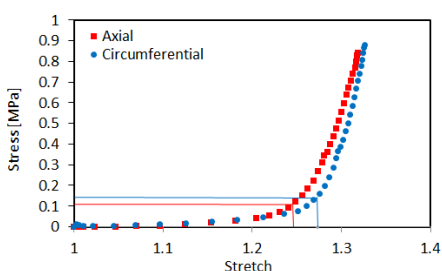

(e) patient 5

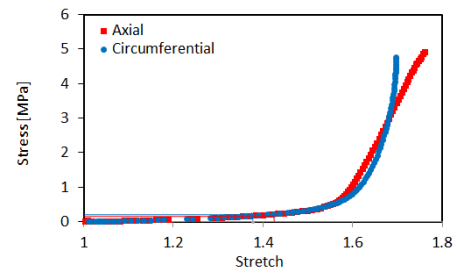

(h) patient 8

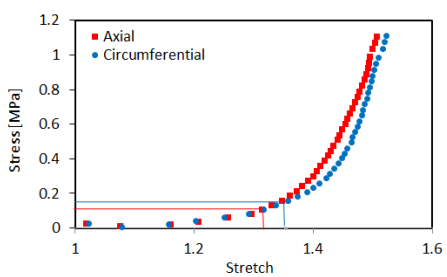

(k) patient 11

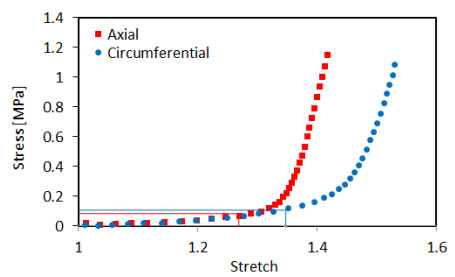

(c) patient 3

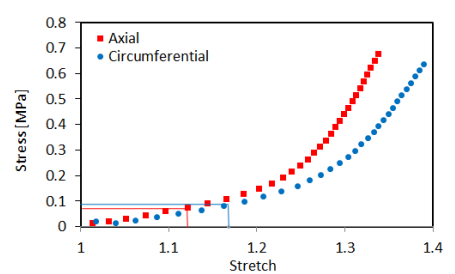

(f) patient 6

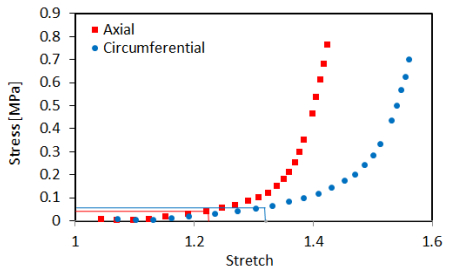

(i) patient 9

Figure 3: Stress-stretch curves obtained from the bulge inflation test for all patients. Solid lines show the in-vivo stretch and stress points ${ }^{15,35}$. 


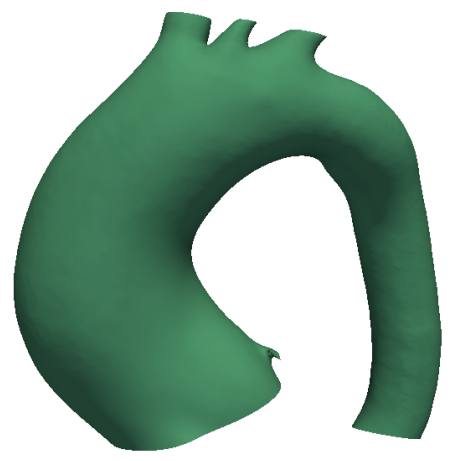

(a) patient 1

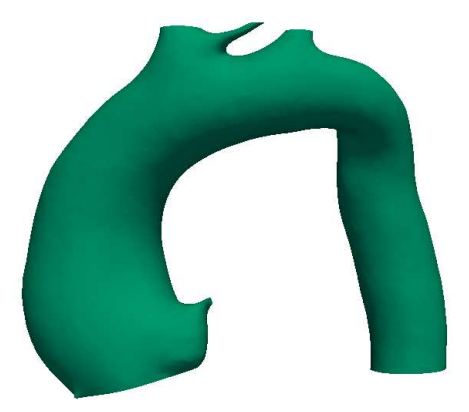

(d) patient 4

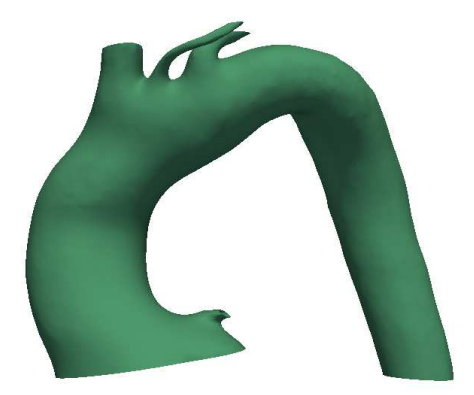

(g) patient 7

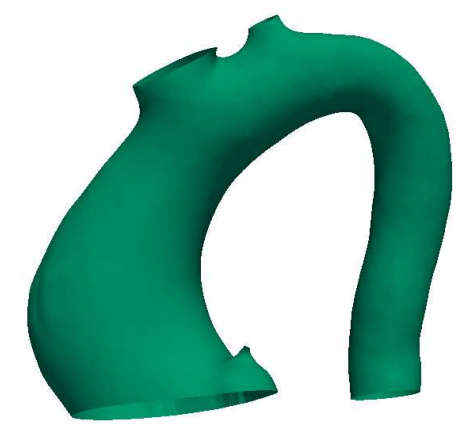

(j) patient 10

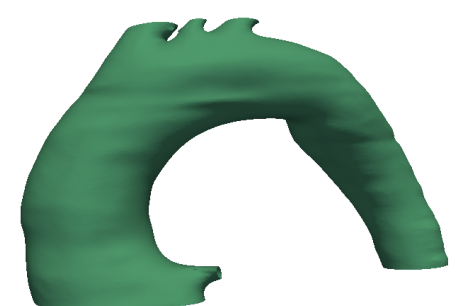

(b) patient 2

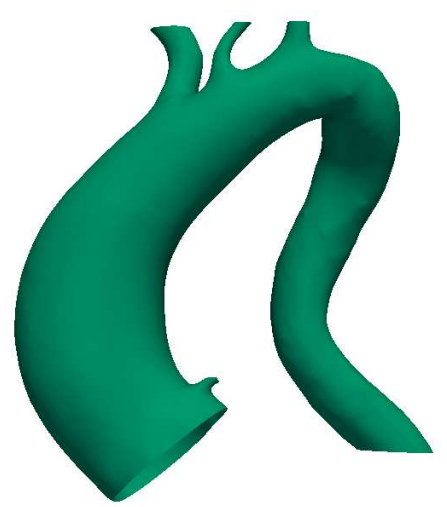

(e) patient 5

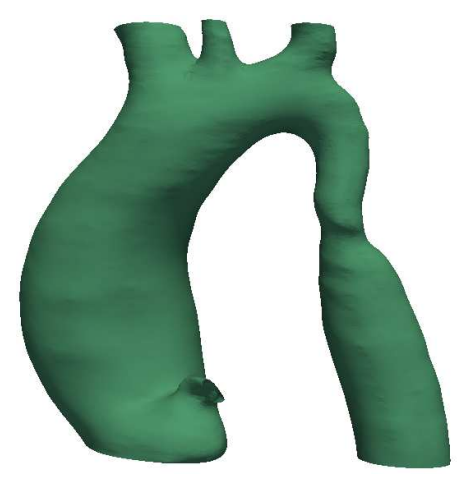

(h) patient 8

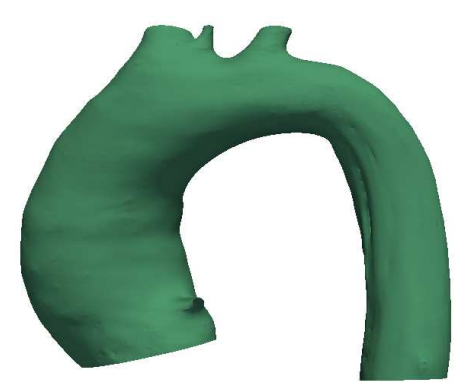

(k) patient 11

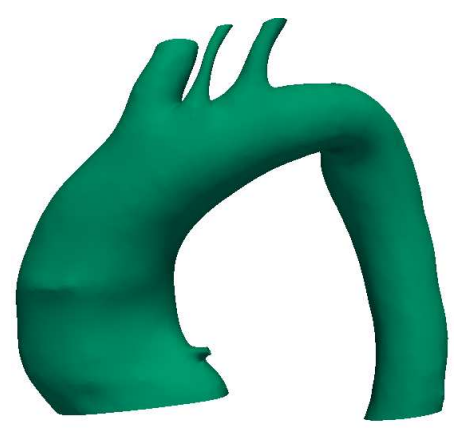

(c) patient 3

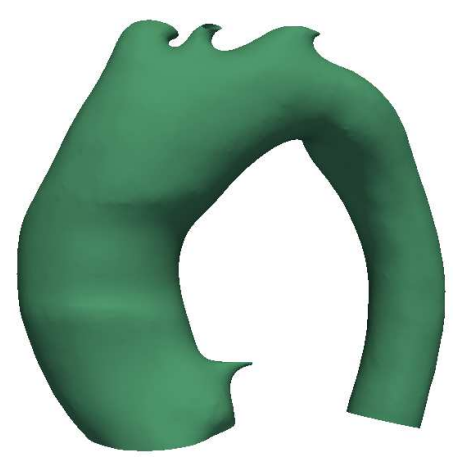

(f) patient 6

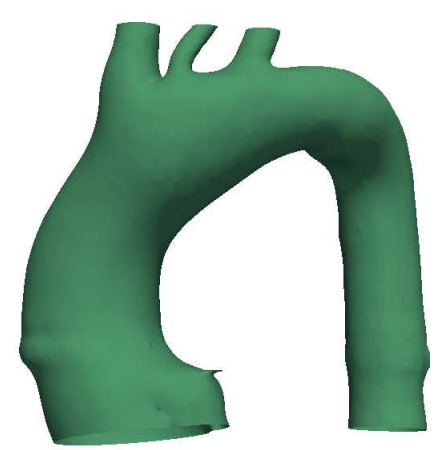

(i) patient 9

Figure 4: Segmented geometries of aorta for diastole using CT images for 11 patients. 


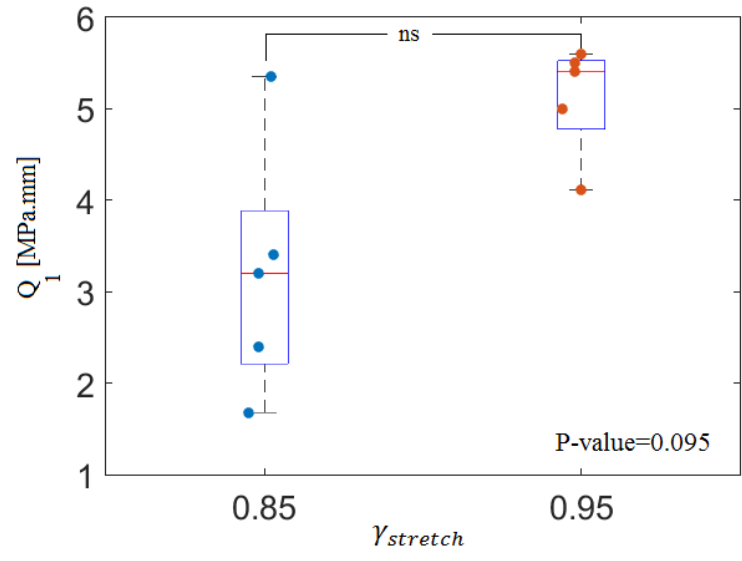

(a)

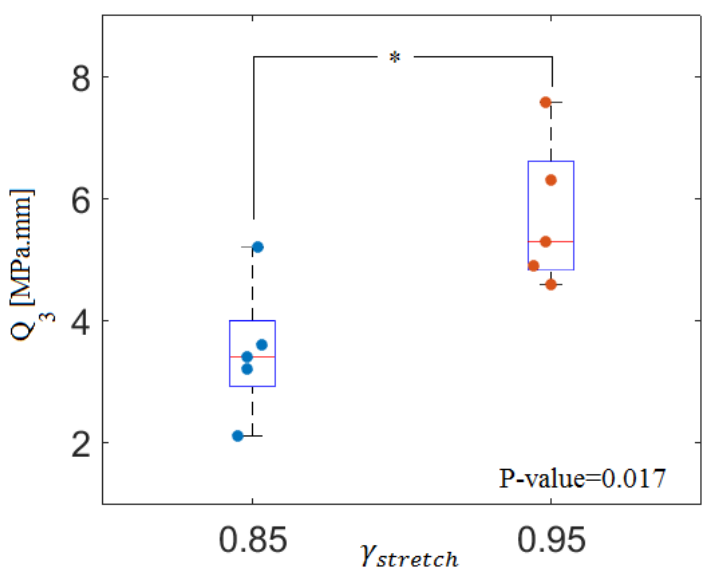

(c)

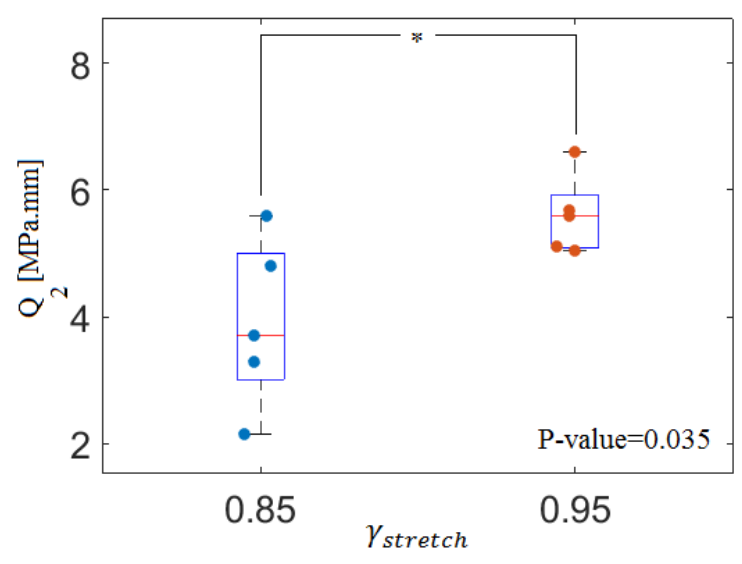

(b)

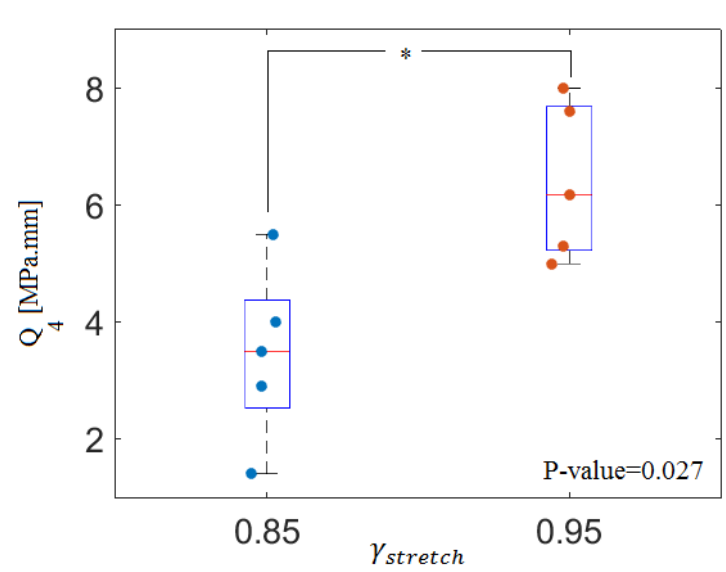

(d)

Figure 5: Boxplots of both groups of patients a- $Q_{1}$ (median value across ATAA region 1), b- $Q_{2}$ (median value across ATAA region 2), c- $Q_{3}$ (median value across ATAA region 3 ) and d- $Q_{4}$ (value at center of ATAA). 


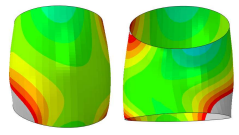

(a) patient 1
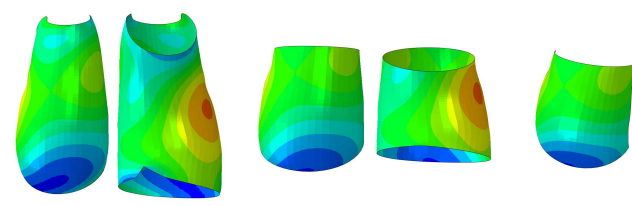

(c) patient 3
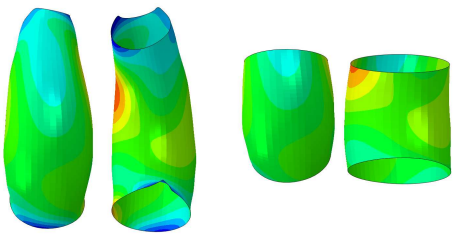

(e) patient 5
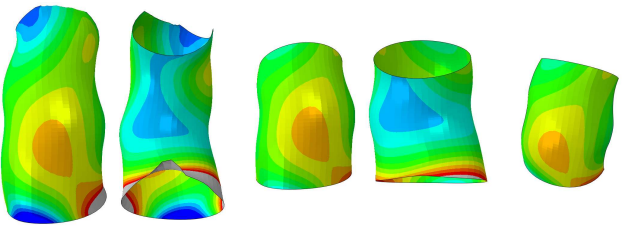

(g) patient 7
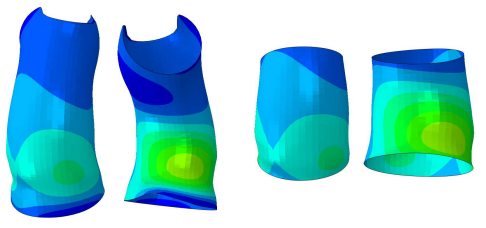

(i) patient 9
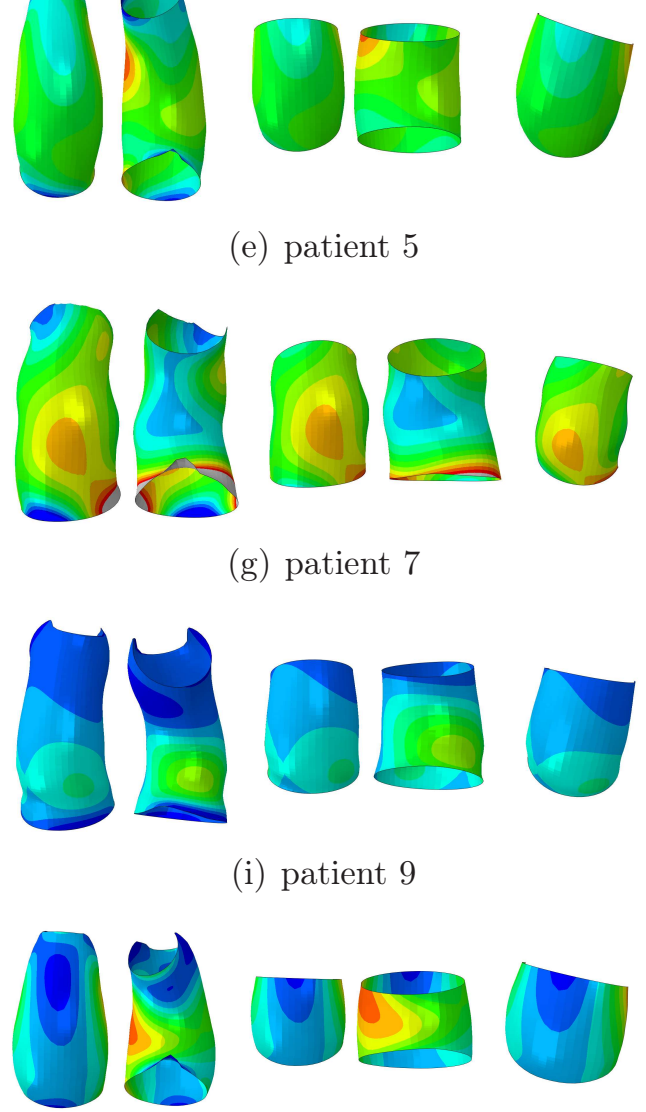
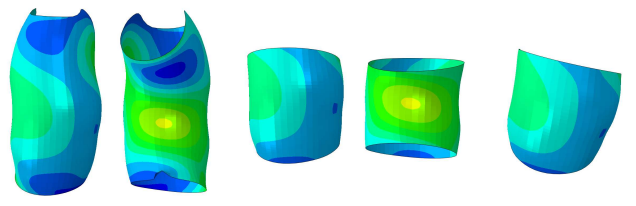

(f) patient 6
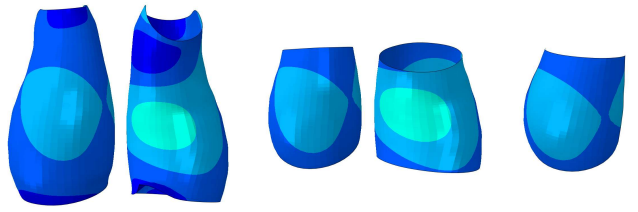

(h) patient 8
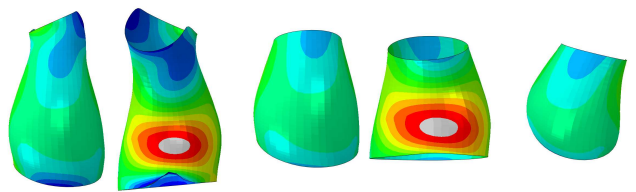

(j) patient 10

(k) patient 11

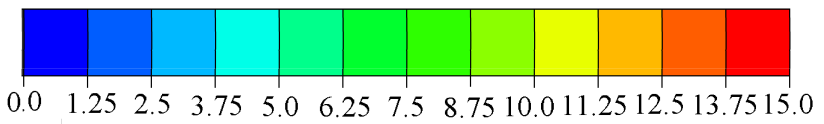

Figure 6: Extensional stiffness distribution [MPa.mm] obtained with the LESI methodology across the ascending thoracic aorta of ten patients. From left to right, region 1 (frontal and distal views), region 2 (frontal and distal views) and region 3. 


\section{List of Tables}

1 Clinical and biomechanical parameters of the 11 patients. . . . . . . . . . 30

2 Median values of extensional stiffness identified in the different ATAA regions 31

3 Pearson product-moment coefficient values . . . . . . . . . . . . 32 
Table 1: Clinical (sex, age and pressure difference) and biomechanical (stretch ratio risk criterion, rupture stress, in - vivo stiffness ${ }^{35}$ and in - vitro stiffness ${ }^{15}$ ) parameters of the 11 patients.

\begin{tabular}{lllllllll}
\hline $\begin{array}{l}\text { Patient } \\
\text { ID }\end{array}$ & Sex & Age Valve phenotype & $\Delta P[\mathrm{kPa}]$ & $\gamma_{\text {stretch }}$ & $\begin{array}{l}\sigma_{\text {rup }} \\
{[\mathrm{MPa}]^{15}}\end{array}$ & $\begin{array}{l}E_{\text {in-vivo }} \\
{[\mathrm{MPa} . \mathrm{mm}]^{35}}\end{array}$ & $\begin{array}{l}E_{\text {in-vitro }} \\
{[\mathrm{MPa} . \mathrm{mm}]^{15}}\end{array}$ \\
\hline \hline 1 & M & 58 & BAV & 8.66 & 0.87 & 0.79 & 7.199 & 1.216 \\
2 & M & 78 & TAV & 5.34 & 0.92 & 1.12 & 2.723 & 2.202 \\
3 & M & 61 & BAV & 11.5 & 0.88 & 1.11 & 5.436 & 0.922 \\
4 & M & 69 & TAV & 5.34 & 0.84 & 0.50 & 1.669 & 0.866 \\
5 & M & 70 & BAV & 5.8 & 0.95 & 0.90 & 2.689 & 3.996 \\
6 & M & 81 & TAV & 5.34 & 0.84 & 0.65 & 2.967 & 1.237 \\
7 & M & 84 & TAV & 13.4 & 0.94 & 1.24 & 8.189 & 3.314 \\
8 & M & 27 & BAV (Marfan) & 8.66 & 0.81 & 4.84 & 2.062 & 0.569 \\
9 & M & 77 & TAV & 10.33 & 0.94 & 0.78 & - & - \\
10 & F & 78 & TAV & 5.34 & 0.95 & 1.31 & 10.089 & 4.124 \\
11 & M & 57 & BAV & 5.34 & 0.89 & 0.98 & 3.999 & 1.271 \\
\hline
\end{tabular}


Table 2: Median values of extensional stiffness identified in the different ATAA regions. $Q_{1}, Q_{2}, Q_{3}$ and $Q_{4}$ are the extensional stiffness of the whole ascending thoracic aorta, the segment of ascending thoracic aorta which was removed during the surgical procedure, the square sample of $40 \times 40 \mathrm{~mm}$ which was cut for the bulge inflation test and the center of this square (center of the outer curvature part of the ascending thoracic aorta) respectively.

\begin{tabular}{lllll}
\hline $\begin{array}{l}\text { Patient } \\
\text { ID }\end{array}$ & $Q_{1}[\mathrm{MPa} . \mathrm{mm}]$ & $Q_{2}[\mathrm{MPa} . \mathrm{mm}]$ & $Q_{3}[\mathrm{MPa} . \mathrm{mm}]$ & $Q_{4}[\mathrm{MPa} . \mathrm{mm}]$ \\
\hline \hline 1 & 7.48 & 8.3 & 8.7 & 10.7 \\
2 & 5 & 5.1 & 4.9 & 5 \\
3 & 5.35 & 5.6 & 5.2 & 5.5 \\
4 & 2.39 & 3.3 & 3.2 & 2.9 \\
5 & 5.6 & 6.6 & 6.3 & 7.6 \\
6 & 3.2 & 3.7 & 3.4 & 3.5 \\
7 & 5.41 & 5.69 & 7.59 & 8 \\
8 & 1.68 & 2.15 & 2.11 & 1.41 \\
9 & 5.5 & 5.6 & 5.3 & 5.3 \\
10 & 4.11 & 5.04 & 4.6 & 6.17 \\
11 & 3.4 & 4.8 & 3.6 & 4 \\
\hline
\end{tabular}


Table 3: Pearson product-moment coefficient value, $\rho$, between stiffness of different ATAA regions and in-vitro parameters.

\begin{tabular}{llllllll}
\hline & $\gamma_{\text {stretch }}$ & $\begin{array}{l}E_{\text {in-vitro }} \\
{[\mathrm{MPa} . \mathrm{mm}]}\end{array}$ & $\begin{array}{l}\sigma_{\text {rup }} \\
{[\mathrm{MPa}]}\end{array}$ & $\begin{array}{l}Q_{1} \\
{[\mathrm{MPa} . \mathrm{mm}]}\end{array}$ & $\begin{array}{l}Q_{2} \\
{[\mathrm{MPa} . \mathrm{mm}]}\end{array}$ & $\begin{array}{c}Q_{3} \\
{[\mathrm{MPa} . \mathrm{mm}]}\end{array}$ & $\begin{array}{c}Q_{4} \\
{[\mathrm{MPa} . \mathrm{mm}]}\end{array}$ \\
\hline \hline$\gamma_{\text {stretch }}$ & 1 & 0.92 & 0.64 & 0.84 & 0.88 & 0.82 & 0.89 \\
$E_{\text {in-vitro }}$ & 0.92 & 1 & 0.62 & 0.63 & 0.72 & 0.74 & 0.88 \\
[MPa.mm] & & & & & & & \\
$\sigma_{\text {rup }}[\mathrm{MPa}]$ & 0.64 & 0.62 & 1 & 0.54 & 0.54 & 0.57 & 0.64 \\
$Q_{1}[\mathrm{MPa} . \mathrm{mm}]$ & 0.84 & 0.63 & 0.54 & 1 & 0.95 & 0.9 & 0.88 \\
$Q_{2}$ [MPa.mm] & 0.88 & 0.72 & 0.54 & 0.95 & 1 & 0.87 & 0.91 \\
$Q_{3}[\mathrm{MPa} . \mathrm{Mm}]$ & 0.82 & 0.74 & 0.57 & 0.90 & 0.87 & 1 & 0.95 \\
$Q_{4}[\mathrm{MPa} . \mathrm{mm}]$ & 0.89 & 0.88 & 0.64 & 0.88 & 0.91 & 0.95 & 1 \\
\hline
\end{tabular}

